Fecha de recepción: marzo 2021 Fecha de aceptación: abril 2021 Versión final: mayo 2021

\section{El arte y la diversidad funcional: Nuevos sistemas digitales desde la creación y para la creación}

Marta Rico Cuesta ${ }^{(1)} y$

Guadalupe Durán Domínguez ${ }^{(2)}$

Resumen: Las tecnologías, a lo largo de la historia, han sido empleadas para mejorar las habilidades y rendimiento de las diferentes acciones o actividades que se plantean las personas; pero este valor se incrementa cuando el individuo prescinde o tiene dificultades para utilizar alguna/s parte/s de su cuerpo; ya sea por dificultades funcionales, anatómicas o fisiológicas, o venga dado por un fallo neuronal que afecte a una, varias o todas las acciones que el ser humano pueda desarrollar. Ante esto, el arte puede ser un vehículo de expresión que canaliza sentimientos y emociones, que visibiliza los diferentes modos de vida y ayuda a mejorarlos; favoreciendo la inclusión y modificando la imagen, en ocasiones estereotipada, que recae sobre las personas con diversidad funcional.

Con la llegada de la era digital e internet, las herramientas binarias se han vuelto imprescindibles para toda persona con capacidad económica. En determinados casos, la propia intervención a través de redes sociales, en la web 2.0 , ha facilitado la conexión entre e individuos con otras necesidades; reactivando una nueva tipología de mecenazgo activo que interviene directamente sobre la capacidad física de crear, e incorporando múltiples opciones de visualización, difusión, y puesta en valor.

Para ello, nos servimos de la exploración y observación de diferentes casos de artistas con diversidad funcional, como esclerosis lateral amiotrófica (ELA), esclerosis múltiple (EM) o hemiplejia; que han estudiado formas de comunicación gracias a neurosensores ( $\mathrm{y}$ otras formas artísticas derivadas de los mismos), describiendo otras tipologías de estructuras digitales y tecnológicas únicas, pero con la posibilidad de propiciar una fabricación de carácter industrial, y para facilitar otros sistemas para la comunicación en los medios de expresión habituales (visual, auditivo, ...).

En este estudio se focaliza nuestra atención en las ramas artísticas y el mundo del arte, y en cómo la diversificación digital ha influenciado a la narración artística; permitiendo mantener la capacidad creadora, entendida como la acción de crear arte objetual en individuos que ven modificadas sus habilidades motoras.

Se parte de un enfoque feminista para abordar las identidades no normativas, puesto que éste se compromete con la diferencia; entendiendo el cuerpo como elemento sobre el que recae el poder y que puede ejercer resistencia. Así lo expresa el trabajo de Moon Ribas cuando modifica su cuerpo con la ayuda de la tecnología, introduciendo un nuevo órgano que la capacita para percibir las ondas sísmicas de la tierra. Se exponen así, propuestas de creación y expresión artística de individuos, cuyo interés artístico y su capacidad cognitiva por la creación se intensifican, y/o cómo se han servido de estas herramientas coetáneas para fundamentar otras estéticas y conceptos artísticos; ofreciendo nuevos puntos de vista sobre la creación, y proponiendo otras maneras de abordar el proceso creador. Esto 
motiva cambios en el propio estado del arte; propiciando, dentro de la propia estructura binaria, otros soportes de creación para la difusión e incluso la propia financiación.

Se pretende aportar un estudio innovador al relacionar nuevos modos de vida en el mundo del arte: de expresión y de creación para los individuos con diversidad funcional, que utilizan nuevos mecanismos tecnológicos, que propician y motivan sus acciones, y que a su vez, les sirven como sistemas de difusión y financiación estrategias transmedia, tanto para la creación como para la comercialización.

Palabras clave: Arte - Diversidad funcional - Diseño Industrial - Era digital - Web 2.0, Proceso creativo - Internet - Tecnología - Cyborg - Sensores.

[Resúmenes en inglés y portugués en las páginas 165-168]

(1) Marta Rico Cuesta es Doctora en Historia y Artes por la Universidad de Granada y Profesora del área de Dibujo en el Grado en Ingeniería en Diseño Industrial y Desarrollo de Producto de la Universidad de Extremadura. Es productora e investigadora artística, destacando la exposición individual "La habitación del cuerpo" (2013) en la Fundación Santander Creativa (Cantabria, España) y las exposiciones colectivas "Mappae Mundi" (2011) en la Fundazione Orestiadi (Palermo, Italia), "Reminiscencias Lumínicas" en el Centro Federico García Lorca dentro del Festival de Cine Clásico de Granada Retroback, y "El peso del Alma" (2017). Es miembro del Grupo de Investigación HUM-425 de la UGR, donde se dedica al análisis de los lenguajes artísticos contemporáneos que intervienen y cuestionan las políticas culturales de representación e inclusión social.

(2) Guadalupe Durán Domínguez es Doctora en Actividades Artísticas por la Universidad de Extremadura (UEX) y profesora en el área de Dibujo en la UEX (España) desde 2008. Responsable del Aula de Fotografía del Vicerrectorado de Extensión Universitaria de la UEX entre 2006 y 2016. Ha comisariado los certámenes "Otoño Fotográfico de Extremadura" (2006-2012) y "FotoExtremadura" (2009 y 2013). Como artista, destaca la participación en las exposiciones "Objetivo: Cáceres 2016" (Bruselas, 2008), "Nómadas" (Itinerante, 2008), en "FotoSur" - Feria de Arte ForoSur- (Cáceres, 2007-2011) y "Enlazados” (Badajoz, 2017).Trabaja en el grupo de investigación La creación artística: Música, imagen y movimiento, participando en múltiples proyectos de Investigación y contratos vinculados al arte digital, y publicando artículos internacionales con esta temática. 


\section{Introducción}

Estamos inmersos en plena era de la tecnología, donde estamos rodeados de infinidad de dispositivos que empleamos en nuestro cotidianeidad, en el hogar, el trabajo, los desplazamientos, etc. la mayoría de ocasiones con la justificación de que nos facilitan nuestro día a día. Es tanto el uso que hacemos de estos dispositivos y lo que nos puede mejorar nuestra vida, que en ocasiones se convierten en una extensión de nuestro propio cuerpo o incluso una parte de éste, haciendo que se modifique la forma que tenemos de entender el cuerpo. Además, las tecnologías hacen que tengamos nuevas capacidades o incrementemos las ya existentes. Estas características de las tecnologías provocan que las personas con diversidad funcional las empleen para modificar sus condiciones.

En este texto, abordaremos una reflexión de cómo desde el arte se han empleado los nuevos sistemas digitales en las personas con diversidad funcional. Unas prácticas que no debemos de entender de forma aislada, sino en relación con el contexto, la economía, la política... Por ello, estamos obligados a destacar que este estudio se enmarca dentro de la sociedad occidental, en la que rigen los cuerpos sanos, atléticos, jóvenes y delgados, y aquellos que no obedezcan a estos patrones se considerarán cuerpos no normativos, con escasa representación y expulsados del sistema. Esto les sucederá a las personas enfermas $o$ las personas con diversidad funcional.

Una investigación cuyo marco temporal vendrá comprendido desde finales del siglo XX hasta la actualidad, más concretamente a partir de los años noventa, cuando hay una gran expansión de las tecnologías, lo que generará teorías como las de Gergen (1997) sobre la identidad múltiple y la fragmentación del sujeto, las de Stone (1996) con el espacio virtual y el hombre-máquina, o más actuales como la de Sloterdijk (2008) con el poshumanismo. A partir de esta década "temas y preocupaciones como la personalización de lo político, el (bio)cuerpo como campo de batalla y la vuelta a la performance, la crisis y la reformulación de las identidades y el género/sexo, las nuevas tecnologías o la visibilidad en el espacio público y en torno a la redefinición del trabajo (producción/reproducción)...toman protagonismo para múltiples personas feministas, dentro de la escena del pensamiento y las imágenes" (Navarrete, Ruido y Vila, 2005, p.172)

Un feminismo (Butler, 2007; Platero Méndez, 2012; Sandoval, 2004) que va a constituir un pilar fundamental para nuestro estudio, pues partimos de este enfoque para abordar las identidades no normativas, puesto que éste se compromete con la diferencia (Brah, 2004); entendiendo el cuerpo como un elemento sobre el que recae el poder y que puede ejercer resistencia. Se pretende cuestionar el sistema hetero-patriarcal hegemónico y mostrar otras realidades, puesto que este va en detrimento no sólo de las mujeres, sino también de las identidades que quedan fuera de lo considerado como normativo. Movimiento al que podemos vincular, por algunas luchas comunes, el activismo queer (Córdoba, Sáez, y Vidarte, 2005) que cuestiona los planteamientos biologicistas apoyados por los dispositivos científicos que naturalizan el sexo y hacen de la heterosexualidad la norma; y el cripple (McRuer, 2006) que como apunta Preciado (2011), la construcción cultural de la discapacidad tiene puntos en común con la masculinidad, el discurso médico, la reproductividad y con las luchas intersexuales y transexuales. Este último movimiento, el cripple, constituye la base fundamental para nuestro estudio al apostar por la visibilización de los 
procesos tecnológicos que nos construyen y centrarse en la disfunción. Por tanto, este enfoque feminista se apoyará en una metodología cualitativa, crítica y comparativa a través del análisis de diversas prácticas de artistas que emplean la tecnología para incrementar las capacidades de las personas con diversidad funcional. Un estudio que partimos desde la interdisciplinariedad, aunando materias de diferentes ámbitos como el arte, la tecnología, la sociología, etc. diluyéndose las fronteras entre las disciplinas.

Fruto de esa interdisciplinariedad son las diversas fuentes en las que nos hemos apoyado para la realización de nuestro estudio, como: Origen, evolución y estado actual del activismo digital y su compromiso social (2017) de García-Estévez para el análisis de la tecnología; Ciencia, Cyborgs y mujeres (1995) de Haraway, Crip Theory (2006) de McRuer, Cojos y precarios haciendo vidas que importan (2022) de Agulló et al. y ¿La muerte de la clínica? (2013) de Preciado, para abordar los cuerpos con diversidad funcional; Arte en la red (2004) de Carrillo y Arte e identidad en Internet (2008) de San Cornelio, entre otros, para abordar las prácticas artísticas. Así como prácticas tecnológicas como el proyecto de Martínez Oliva (2020) o los últimos software desarrollados por empresas como TallK (2021) de Samsung. Aunque sigue surgiendo nueva bibliografía en la que apoyarnos al tratarse de un ámbito de estudio latente y en constante transformación, puesto que constituye un tema actual y se presenta constantemente en evolución porque se sigue avanzando en el campo de las tecnologías, perpetuamente surgen nuevas prácticas, formas de aplicarlas, etc.

\section{El cuerpo no normativo y la tecnología}

"(...) nothing in man -not even his body- is sufficiently stable to serve as a basis for self-recognition or for understanding other men”./[“(...) nada en un hombre- incluso su cuerpo- es suficientemente estable para servir como base del auto-reconocimiento o para el entendimiento de otros hombres"] (Foucault, 1990, p.153).

Foucault nos recuerda que el cuerpo se transforma, no es estable, sino que está sujeto a modificaciones, dado que debemos de entenderlo como un elemento sobre el que recaen las construcciones culturales, lo que provocará que adquiera comportamientos, posturas, etc. establecidos socialmente. Una construcción cultural y social del cuerpo que genera un ideal, una imagen canónica de cómo debe de ser éste, que vendrá marcado por el sistema hetero-patriarcal y capacista en el que estamos inmersos. Este sistema hará que debamos mostrar una perfecta integración dentro del sistema y que aquellos cuerpos que no se adapten sean expulsados y señalados como diferentes. Por tanto, los cuerpos con diversidad funcional se verán obligados a habitar en los márgenes. Una exclusión que se agudiza en su construcción por la interseccionalidad (Platero Méndez, 2012), al ser atravesada por diversas estructuras de opresión y desigualdad como el género, la raza, la orientación sexual, la religión, la nacionalidad, la edad, la situación laboral, familiar, etc.

Así, en este texto, nos centraremos principalmente en analizar qué sucede con las prácticas que emplean la tecnología en los cuerpos en los que experimentan esa exclusión por género (Butler, 2007) y capacitismo. Aunque, no debemos olvidarnos que sobre la diversidad funcional recae un imaginario que dibuja al hombre como un sujeto sin género o femini- 
zado, desprovisto de virilidad al no contemplarlo como un cuerpo productivo y reproductivo (Preciado, 2011). Pues como ya apuntase Butler (2002, p.36) las categorías identitarias nos constriñen y limitan, aunque son necesarias para el feminismo al crear una política de identidad. No obstante, en los ejemplos en los que nos apoyaremos mayoritariamente a lo largo de este trabajo vendrá dado por mujeres con diversidad funcional, al interesarnos analizar qué pasará con esta doble interseccionalidad. Nos interesa detenernos en qué sucede cuando el sujeto además de mujer, tiene diversidad funcional.

Una diversidad funcional con escasa representación en la sociedad y a la que se le vinculan conceptos negativos. Unos imaginarios que no tienen nada de inocuos, sino al contrario; la cantidad de narrativas y discursos que surgen en torno a la diversidad funcional, condicionan su representación, visibilidad, cómo nos relacionamos y convivimos con ella, etc. lo que coarta su aceptación social.

Una aceptación a la que nos puede ayudar la tecnología al mostrar otros cuerpos y/o realizar modificaciones en éstos que alteren o implementen nuestras condiciones, transformando la imagen que tenemos de los cuerpos con diversidad funcional. Un cuerpo que cada vez cobra más importancia, al contrario de lo que parecía y se pronosticaba inicialmente con el surgimiento de la tecnología y el ciberespacio, al estar inmersos en la era de los signos y ser éste el signo por excelencia (San Cornelio, 2008; Carrillo, 2004). Ya que la tecnología ha hecho que entendamos y nos relacionemos con el cuerpo de otras maneras, y por extensión, esto ha influido en las cuestiones identitarias. Así, surge el movimiento cripple en oposición a la normalización y hegemonía capacitistas, que cuestiona las técnicas biopolíticas y visibiliza los procesos tecnológicos que los configuran (Guzmán Castillo y Toboso Martín, 2010). Este movimiento pretende empoderar nuestra situación como personas con diversidad funcional, con nuestro propio espacio y sin la necesidad de realizar modificaciones corporales que nos puedan ayudar a modificar nuestras capacidades o condiciones. Sin embargo, desde otros posicionamientos, se apostará más por el empleo de la biotecnología, la tecnología que se asocia con la medicina para transformar determinadas situaciones. Unos cuerpos cyborgs que subvierte el sistema hegemónico y las clasificaciones que éste genera (Haraway, 1995, p.281).

"Las políticas cyborg implican modelar los mecanismos de poder/conocer que constituyen los sujetos postmodernos, lo cual incluye el luchar por el despliegue de una epistemología de la parcialidad. Pero también requiere una conciencia de los nuevos límites que están emergiendo dentro de una realidad marcada por la cibernética (...)" (Haraway, 1995, pp.16-17)

Este es el caso de la artista Moon Ribas y la noción que tiene del cuerpo, de su cuerpo, transformándolo al implantarse en los pies sensores sísmicos que le permiten percibir los movimientos tectónicos que están teniendo lugar en cualquier parte del mundo en ese mismo momento y que ella interpreta mediante la danza. Una forma para ella de establecer un mayor vínculo con el planeta y lo que a este le sucede, entender la realidad. O propuestas como la de Miriam Simun y Miriam Songster en Ghostfood (2013) al emplear un dispositivo directo de estimulación olfativa como un empleo distópico en la alimentación. 


\section{Arte, tecnología y diversidad funcional: La tecnología al servicio de la diversidad funcional}

¿Qué es lo que nos hace identificarnos con quienes somos y qué nos hace ser nosotros mismos? El concepto propio de ser humano no viene dado por las capacidades físicas que generen acciones o nos permitan comportarnos como queremos o estamos acostumbrados; si bien, de ahí el capacitismo del ser humano perfecto.

El arte digital nos sumerge en otras identidades diferentes, nos muestra otros espacios para sugerirnos cambios de nuestros estadios; así en "Huit phases de l'illumination" se nos sugiere la posibilidad de irrumpir en nuestro espacio siendo nosotros, los que conocemos, pero privados de todas las capacidades sensitivas que en nuestro día a día nos permiten enfrentarnos e interactuar con el mundo que nos rodea en un juego de realidad mixta, bajo la apariencia de intervención artística, que se expuso en el Palacio de Tokyo de París. Con sensores de diferente tecnología audiovisual nos capacitan, o más bien, imposibilitan la práctica del tiempo, el espacio, el sonido, el color, la forma, la profundidad y la velocidad, propios en el cuerpo físico entendido como completo, para provocar otro sentido de identidad en un universo virtual paralelo al nuestro (Aramique et al., 2015).

De ese juego de identidad nos hace partícipes Patricia Lay-Dorsey (BBC-mundo, 2014), cuando esta artista en silla de ruedas, nos muestra sus reflexiones a través de sus autorretratos. Tantas búsquedas de su propio yo, de su propia identidad, destacando quién se consideraba ella misma antes, y quién puede ser ahora, cuando no encuentra diferencias reales que le hagan creerse/crearse personas verdaderamente diferentes. No existe una modificación del individuo, cuando sí, una variación en su modelo de ejecutar y de relación con sus actividades y su contexto, aunque marcado por la interseccionalidad. Ante esto, marca justamente su humanidad, como una muestra de exaltación de su propio yo a través del uso de la cámara digital, en el propio proceso de creación y de exhibición a través de su página web (Lay-Dorsey, 2021), así como otros sistemas transmedia para la difusión de los resultados.

Pero además, el arte le permite expresarse, le permite relacionarse e interactuar tanto con su entorno próximo como con el más lejano; en la misma dirección como la reconocida Yayoi Kusama, que utiliza su medio para organizar en su entorno sus patrones de vida, recreando aquellas visiones o alteraciones visuales y auditivas que le siguen desde niña, plagándolo todo de puntos en su aportación sobre el espacio con reflejos de redes infinitas. Resuelve así su trastorno obsesivo compulsivo en una búsqueda por la supervivencia (Medina, 2013, pp.13-21).

En esa misma búsqueda de la supervivencia, citamos a la artista Fina Miralles, española, feminista y conocida por su obra conceptual en el land art. Comenzó su andadura de la época franquista y hoy utiliza la expresión artística como autoconocimiento y terapia curativa, tal y como manifiesta en su acción "El bautismo" de 2012, en cuya obra la artista reflejaba la motivación para continuar creando: el propio arte como elemento curativo (Cabré, 2020) y que hace visible en la línea del pensamiento de Patricia Lay-Dorsey, con su última exposición "Soy todas las que he sido", que se ha visualizado hasta el 5 de abril de 2021 en el Museo de Arte Contemporáneo de Barcelona (MACBA). 
Así mismo, el descubrimiento de alguna enfermedad irrumpe en todo proceso creativo, y genera modificaciones de patrones vitales, como en el caso de Laura Harris, que trabaja mosaicos de diferentes tipologías, potenciando materiales reciclados en cada tesela específica para cada espacio vacío. Sus iconos muestran tecnología incrustada a cada realidad visual conocida y se exhiben en la Galería de Bellas Artes de Eggert, North Lake (Wisconsin) y en la Galería de Bellas Artes de Monumentos, Wauwatosa (Wisconsin). Ahora además, utiliza su propia galería virtual en melonheadgallery para difundir las imágenes de su medio de expresión (Harris, 2021), donde es igual a los demás. No olvidemos que las pantallas nos hacen legítimamente iguales; en internet las diferencias desaparecen, nos hacemos iguales, porque lo realmente interesante o importante permanece (Ogbomon, 2014, p. 28).

... Y causan paradigma/s en toda enfermedad degenerativa inmersa en el proceso artístico evolutivo. Como en el caso de la artista Judy Ross Brown en cuyas actividades creativas realistas ha ido interviniendo con una parte diferente de su cuerpo: mano, pie, boca... para determinar en su última declaración en 2009 (Lorente, 2019) su iniciativa por el empleo de la tecnología, en donde, y tal como cita ella textualmente: "Ciertamente me devolvió un sentido de orientación y algo de independencia”.

Es en este sentido como la tecnología encauza nuevas reacciones en el cuerpo, permitiendo actitudes y aptitudes perdidas, en un acercamiento desde las necesidades, por la merma de capacidades en la disfunción degenerativa. En nombre de la Asociación de Esclerosis Múltiple del País Vasco, María Ángeles Lara recibió el reconocimiento por Virtualware, como programa idóneo para la rehabilitación virtual accesible para individuos con diferentes enfermedades neurodegenerativas que permite la mejora del equilibrio, la coordinación o determinados patrones de psicomotricidad con la utilización del juego (Evolv, 2015). En este sentido, Anouk Wipprecht creó Agent Unicorn, un adaptador con forma de unicornio para el estudio de ondas P300 que registran reacciones en el entorno y espacios para niños con trastornos por déficit de atención e hiperactividad (“3d, printing \& design", 2020, p. 42).

$\mathrm{Al}$ acercarnos de este modo a la tecnología, también encontramos propuestas de adaptación digital para mejorar o acceder a estrategias para facilitar la comunicación, como el software TALLK de Samsung, basado en eye tracking que permite a los enfermos de ELA hablar y escribir al interlocutor siguiendo el movimiento ocular (Samsung, 2021). En esta línea, se inventó Kissyface (Not Impossible, 2018a) para control y manejo de un ordenador con el movimiento de alguna parte del rostro (la ceja, los labios, los ojos, ...) para personas con alguna lesión de médula espinal que impida el movimiento.

Pero el mundo de la tecnología va mucho más allá ya que "la tecnología adaptativa puede llegar a reducir el impacto de la discapacidad y satisfacer el derecho de la calidad de vida de las personas con necesidades especiales" (Belen_Sandra, 2015). Así, observamos que el mundo del arte, desde aproximadamente desde la segunda mitad del siglo XX, ha mirado hacia los neurotransmisores para medir ondas cerebrales que permitan el control mental de elementos u objetos, como con el uso de Neuroswitch por parte del grupo informal parisino NNN ("La Générale", 2014), quienes empiezan planteando la medición de ondas cerebrales para posibles interacciones con los dispositivos electrónicos. En la orientación de Ariel Garten, que crea en 2012 y comercializa su dispositivo Muse, una diadema que 
permite manifestar emociones en objetos electrónicos y que permite la democratización de capacidades humanas (latribune.fr, 2012; Renaud, 2013), que en 2014 varió hacia un producto dedicado al estudio de ondas cerebrales y su capacidad de interacción para la mejora de la actividad/inactividad para la mejora en el sueño y la meditación (Interaxon Inc., 2014).

Esa utopía inicial de Ariel Garten, la traduce Mick Ebeling, con su proyecto para Tony Quan, famoso grafitero estadounidense, cuya trayectoria artística se coartó al quedarse cuadrapléjico por Esclerosis Lateral Amiatrófica (ELA). Igual que con otros proyectos para el desarrollo de material tecnológico adaptativo que mejore las capacidades funcionales, la fundación Not Impossible Labs, en 2010 crea el Eyewriter, con la finalidad de que Tempt One recupere, no solo aptitudes comunicativas, si no también y sobre todo, su capacidad artística con la utilización de un sensor que permita traducir una intención ocular que un sensor captura, y traslada a un resultado físico real y casi inmediato, mediante una estructura motorizada que recibe la señal directa y que el propio artista visualiza desde su cama mediante una serie de cámaras y pantallas, y describen toda la actividad ( Not Impossible, 2018b).

En este medio, encontramos otro ejemplo más que ha de mencionarse y que se materializa en la exposición "Brain on Fire", de la artista Heide Pfüetzner (Maturana, 2013; Uno Cero, 2013), que presenta una limitación en sus movimientos debido al ELA. Como consecuencia de ello, comenzó una campaña de crowdfunding en Start next para continuar con su trayectoria artística, si bien, con nuevas búsquedas gráfico-plásticas. Los 6.500 dólares conseguidos le permitieron conseguir el equipamiento de neurosensores, que consistía en dos pantallas junto a sensores y electrodos para leer ondas cerebrales (con el software Intendix Painting de la compañía Gtec). La consecución de dicha exposición le llevó alrededor de 6 años, lo cual nos muestra la complejidad del proyecto.

La tecnología nos otorga la recuperación de capacidades perdidas, nos facilita la posibilidad de recuperar nuestro medio de expresión, bajo una reminiscencia artística gráficoplástica, en nuevos contextos de aprendizaje y adaptación, y bajo esta premisa. Pero la tecnología ahora nos brinda oportunidades nuevas para la recuperación, en el ámbito de la edición del genoma por el método de CRISPR (Martínez-Oliva, 2020).

\section{Conclusión}

Con este estudio, y tal y como se ha expresado en el documento, el análisis comparativo de las diversas prácticas tecnológicas empleadas por y para personas con diversidad funcional nos ha permitido observar cómo se producen una serie de tácticas vinculadas a la diversidad funcional, cada vez más frecuentes, en las cuales destacan las cualidades del arte: la búsqueda del yo, el resarcimiento en el autoconocimiento y exaltación de su propio yo, el reconocimiento en la propia acción y en la terapia, etc.

Y de otro modo, si cabe con mayor protagonismo, destacaremos la tecnología y sus características, principalmente con la aptitud para ayudarnos a modificar nuestras capacidades: ya sea facilitando la comunicación, la expresión artística, la experimentación a partir de 
nuevos medios, y sobre todo, otras estrategias para la exhibición, con galerías digitales online, y la consecución de los objetivos, nuevos o pasados, que retratan a los mismos individuos, esos que hemos sido siempre.

Por tanto, con este estudio surge una clasificación de las prácticas artísticas, pudiendo hablar de dos grandes vías de actuación en torno a las que emergerán subvías, las que el arte y sus características tiene un papel fundamental, y las que se sirven de las cualidades de las tecnologías para producir una mejora o beneficio en las personas con diversidad funcional.

Junto a esto, somos conscientes de que Internet omite la crítica por elementos o capacidades que en ese medio, figuran ausentes. Y si seguimos el vínculo social que genera, no podemos omitir que la red propicia la obtención o financiación de proyectos personales, como proyecto financiado por entidades sin ánimo de lucro, o mediante crowdfunding.

Unas prácticas que en la mayoría de los casos pretenden la mejora de las condiciones y que se produzca una inclusión de las personas con diversidad funcional. Pero también somos conscientes de que en ese empleo de la tecnología para modificar nuestras condiciones corporales, estamos de algún modo reforzando la ocultación de las enfermedades, el dolor, el envejecimiento, la muerte, etc. pudiendo generar el efecto contrario y reforzar la exclusión de las personas con diversidad funcional y trazar una diferenciación con aquellas que no han modificado sus capacidades o su condición mediante el uso de la tecnología.

\section{Bibliografía}

3D, printing \& design (2020). Agent Unicorn: El dispositivo en 3D para niños con TDAH. [en línea] (10) pp. 42-43. [en línea] Recuperado el 12 de marzo de 2021 en http:// www.3dprintingdesign.es/revista/10/

Agulló, C.; et al. (2002). Cojos y precarios haciendo vidas que importan. Madrid: Traficantes de sueños.

Aramique; Morgo, M. et al. (2015). Huit phases de l'illumination. París: Palais de Tokio. [en línea] Recuperado el 20 febrero 2020 en http://aramique.com/HUIT-PHASES-DEL-ILLUMINATION

Brah, A. (2004). Diferencia, diversidad y diferenciación. En: Andalzúa, G.; et al. Otras inapropiables: Feminismos desde las fronteras. Madrid: Traficantes de Sueños. pp.107-136.

BBC-mundo (2014). Vivir con esclerosis múltiple: No hace falta seguir siendo invisible. [en línea] Recuperado el 10 marzo 2021 en https://www.bbc.com/mundo/video_fotos/2014/08/140827_video_mujer_discapacitada_esclerosis_lp

Belen_Sandra (2015)._La Robótica y la mejora de la calidad de las personas con discapacidad. [en línea] Recuperado el 24 marzo 2021 en http://tecnicasdeayudaparaladiscapacidad. blogspot.com/.

Butler, J. (2002). Cuerpos que importan: Sobre los límites materiales y discursivos del "sexo". Buenos Aires: Paidós

Butler, J. (2007). El género en disputa: El feminismo y la subversión de la identidad. Barcelona: Paidós. 
Cabré, M.A. (2020). Una chamada llamada Fina Miralles. [en línea] Recuperado el 15 abril 2021 en https://www.m-arteyculturavisual.com/2020/11/10/una-chamana-llamadafina-miralles/

Carrillo, J. (2004). Arte en la red. Madrid: Cátedra.

Córdoba, D.; Sáez, J.; Vidarte, P. (eds.). (2005). Teoría Queer: políticas bolleras, maricas, trans, mestizas. Barcelona: Egales

Evolv (2015). VirtualRehab recibe el reconocimiento de la Fundación Social Teva. [en línea] Recuperado el 13 abril 2021 en https://evolvrehab.com/es/noticias/recibe-reconocimiento-fundacion-social-teva/.

Foucault, M.(1990). Nietzsche, Genealogy, History. En: Rainbow, P. The Foucault Reader. New York: Pantheon Books.

García-Estévez. (2017). Origen, evolución y estado actual del activismo digital y su compromiso social: Ciberactivismo, hacktivismo y slacktivismo. Actas del II Congreso Internacional Move.net. Sevilla: Universidad de Sevilla. pp. 139-156.

Gergen, K (1997). Toward a cultural constructionist psychology. Theory and Psychology, 7, pp.31-36.

Guzmán Castillo, F. y Toboso Martín, M. (2010). Cuerpos, capacidades, exigencias funcionales...y otros lechos de Procusto. Política y sociedad, 1(47):67-83.

Haraway, D. (1995). Ciencia, cyborgs y mujeres. Valencia: Cátedra.

Interaxon Inc. (2018). Interaxon Inc., leader mondial de la technologie de détection cérébrale pour grand public fait part de la disponibilité de son app de méditation Muse $e^{\circledast}$ langue française, allemande et espagnole. [en línea] Recuperado el 21 febrero 2021 en https:// www.prnewswire.com/news-releases/interaxon-inc-leader-mondial-de-la-technologiede-detection-cerebrale-pour-grand-public-fait-part-de-la-disponibilite-de-son-app-demeditation-muse-en-langue-francaise-allemande-et-espagnole-686676391.html

La Générale (2014). Neuroswitch@«The brain»: Daejeon Museum of Art . 201411 22-2015 02 08." [en línea] Recuperado el 12 febrero 2021 http://www.lagenerale.fr/?p=4878

Lay-Dorsay, P. (2021). Photography. [en línea] Recuperado el 20 marzo 2021 en https:// www.patricialaydorsey.com/

Latribune.fr (2012). Un serre - tête connecté qui lit dans vos pensées. [en línea] Recuperado el 20 febrero 2021 en https://www.latribune.fr/journal/edition-du-0512/special-le-web12-paris/735048/un-serre-tete-connecte-qui-lit-dans-vos-pensees.html

Lorente Guerrero, E. (2019). Never say never! by Judy Ross Brown artist living with multiple sclerosis. [en línea] Recuperado el 13 abril 2021 en https://coloringnursing.wordpress. com/2019/10/16/never-say-never-by-judy-ross-brown-artist-living-with-multiplesclerosis/

Martínez Oliva, B., 2020. Crispr, una herramienta para editar genomas. Gaceta Medica Boliviana, 43(2), pp.179-183.

Maturana, J. (2013). Pintar con la mente ya es una realidad, avance del control mediante ondas cerebrales. [en línea] Recuperado el 5 abril 2021 en https://www.xataka.com/ historia-tecnologica/pintar-con-la-mente-ya-es-una-realidad-avance-del-controlmediante-ondas-cerebrales

McRuer, R. (2006). Crip theory: Cultural signs of queerness and disability. New York: New York University Press. 
Medina, F. (2013). Yayoi Kumasa. En VV.AA. (2013). "Al Límite: Diez años de Arte Contemporáneo." Santiago de Chile: Al Límite (13-21)

Navarrete, C.; Ruido, M; y Vila, F. (2005). Trastornos para el devenir: entre artes, políticas feministas y queer en el Estado español. En: Desacuerdos sobre arte, políticas y esfera pública en el Estado español. No2:158-187.

Not Impossible (2018a). Tomorrow: not impossible TEMPT ONE. [en línea] Recuperado el 22 marzo 2021 en https://youtu.be/8xc1zca-SO8

Not Impossible (2018b). Kissyface: A not impossible story. [en línea] Recuperado el 22 marzo 2021 en https://thriveglobal.com/stories/kissyface-a-not-impossible-story/

Ogbomon, J. (2014). Retrato. En Madox, S.(2014). Guía de Recursos sobre la Parálisis. New Jersey: Paralysis Resource Center (28-29).

Platero Méndez, R. L. (2012). Intersecciones: Cuerpos y sexualidades en la encrucijada. Barcelona: Bellatera.

Preciado, P. (2011). El cuerpo impropio. Guía de modelos somatopolíticos y de sus posibles usos desviados. [en línea]. Recuperado el 23 abril 2013 en http://ayp.unia.es/index. php?option=com_content \&task=view\&id=678.

Preciado, P. (2013). “¿La muerte de la clínica?”. En: Preciado, P. Somateca 2013: vivir y resistir en la condición neoliberal". [en línea]. Madrid: Centro de Arte Reina Sofía. Recuperado el 26 de abril de 2013 en https://www.museoreinasofia.es/multimedia/muerte-clinica.

Renaud (2013). Présentation et demostration de Muse, Le serre - tête connecté.[en línea] Recuperado el 20 febrero 2021 en https://objetconnecte.net/presentation-muse/

Samsung (2021). TALLK. [en línea] Recuperado el 22 marzo 2021 en https://www.samsung. com/es/tecnologiaconproposito/accesibilidad-bienestar/TALLK/

San Cornelio, G. (2008). Arte e identidad en Internet. Barcelona: UOC.

Sandoval, C. (2004). Nuevas ciencias: Feminismo cyborg y metodología de los oprimidos. En: Andalzúa, G.; et al. Otras inapropiables: Feminismos desde las fronteras. Madrid: Traficantes de Sueños. pp. 22-39.

Sloterdijk, P. (2008). Normas para el parque humano. (trad.Teresa Rocha). España: Siruela. Stone, A. R. (1996). The war of desire and technology. Cambridge: MIT Press.

UnoCero (2013). Artista paralizada logra pintar con la mente. [en línea] Recuperado el 3 abril 2021 en https://transformacionmovimiento.wordpress.com/2013/07/16/artistaparalizada-logra-pintar-con-la-mente/

Art and differently abled: new digital systems from its creation well as for the creation

Abstract:Througout history, technology has been used in order to improve the skills and performance enhacing of the actions or issues that are arised from any person; but this value is increased when one individual experience dificulties or even get by without a member of the body; either by functional, anatomical or physiological impairments. it can also be very often brought about a failure of the neuronal function that affect only one or several functions that the human being can develope. In view of this, Art could be a 
vehicle for expresing and chanelling feelings and emotions that make the different ways of life visible and it can assist in improving. This mean would favour the integration and alter which in some cases it seems a stereotypical image, and despite everything it usually lies with people with functional disabilities.

With the advent of internet and digital tecnology, binary tools (online trading instruments) have become indispensable for everybody who is economically able. In certain cases, the intervention itself througout social media on the 2.0 web connection between individual with special needs has been provided, relaunchin a new typology of a lively patronage which is first-hand related to the ability of creating and incorporating multiple choices of displaying, dissemination and highlighting its importance.

We deliver through the exploitation and inspection of different cases of artists with functional disabilities such as Amyotrophic lateral sclerosis (ALS), multiple sclerosis (MS) or hemiplegia. These people have studied mans of communication with the help of neuronal sensors and other art forms which are derived thereof, describing other types of digital structures and unique technologies which could contribute to manufacturing in an industrial nature, and also for communication in common means of expresión, as for instance visual means or auditory means, etc.

Our attention is captured by this study on the branch of art and the art scene, and also it is focused on how digital diversification has influenced on our artistic narration, thereby allowing us to maintain our creative potentia, which is understood as the action of achieving object-based art, regarding to people who are aware that their motor skills are being modified.

It stars on a feminist approach to move away from non-normative individuality, since this one makes a commitment to a difference (or remainder). Understanding the human body as an element which bears all the power and may be resisted. This is reflected by Moon Ribas, when she tries to modify her own body with the help of technology, hading a new organ implanted which enables her to feel seismic waves from the earth. So that new proposals to the creation and art expression from human beings are displayed in this way, taking into account the artistic attraction and the cognitive ability regarding to creation is enhanced and how they have made use of these contemporary tools to substantiate some other strategies and artistic conceptions. In this manner, proposals for the creation and artistical self-expresions are displayed, since the artistic attraction and the cognitive ability intensify, and it also shows how they have made use of these contemporary tools to support these strategies and artistic concepts, so they are trying to show a new perspective on the creation and recommending other ways of dealing with a creative process. This could be what helps to motivate changes in the art state itself, providing different suppor creations to be disseminated or even Project funding.

Connecting new life-styles within the art world it attempts to provide a pioneering study: for diffently abled individuals who make use of new technological tools which promote and encourage their actions, and at the same time serves as a dissemination system and also as strategies for transmedia funding for both, creation and marketing.

Key words: Art - Differently abled - Industrial Design - The advent of internet and digital technology - 2.0 web - Creative process - Internet - Technology - Cyborg, Sensors. 


\section{A arte e a diversidade funcional: Interação de novos sistemas digitais no processo criativo}

Resumo: As tecnologias, ao longo da história, foram aplicadas para melhorar as habilidades e rendimento das diferentes ações ou atividades das pessoas. No entanto, este valor aumenta quando o indivíduo prescinde ou tem dificuldade em utilizar alguma/s parte/s do seu corpo, quer por dificuldades funcionais, anatómicas ou fisiológicas quer por um problema neurológico que afete uma, várias ou todas as ações que o ser humano possa desenvolver. Perante este facto, a arte pode constituir um veículo de expressão que canaliza sentimentos e emoções, que torna visível os diferentes modos de vida e ajuda a melhorá-los favorecendo a inclusão e modificando a imagem, muitas vezes estereotipada, que recai sobre as pessoas com diversidade funcional.

Com a chegada da era digital e da internet, as ferramentas binárias tornaram-se imprescindíveis para todas as pessoas com possibilidades económicas. Em certos casos, a própria intervenção através das redes sociais, na web 2.0, facilitou o contacto entre indivíduos com outras necessidades, reativando um novo tipo de mecenato ativo que intervém diretamente sobre a capacidade física de criar incorporando múltiplas opções de visualização, difusão e valorização.

Assim, observámos diferentes casos de artistas com diversidade funcional como esclerose lateral amiotrófica (ELA), esclerose múltipla (EM) ou hemiplegia que estudaram formas de comunicação graças a neuro-sensores (e outras formas artísticas derivadas dos mesmos), descrevendo outras tipologias de estruturas digitais e tecnológicas únicas, mas com a possibilidade de produção industrial e de facilitar outros sistemas para a comunicação nos meios habituais de expressão (visual, auditivo...,).

Neste estudo, o foco da nossa atenção recai por um lado sobre as diferentes formas de expressão artística e o mundo da arte e por outro no modo em que a diversificação digital influenciou a narração artística permitindo manter a capacidade criativa entendida como a ação de criar arte objetual em indivíduos que veem as suas habilidades motoras modificadas.

Parte-se de um ponto de vista feminista para abordar as identidades não normativas, uma vez que este compromete-se com a diferença, entendendo o corpo como um elemento sobre o qual recai o poder e que pode exercer resistência. Assim o exprime o trabalho de Moon Ribas quando modifica o seu corpo com a ajuda da tecnologia introduzindo um novo órgão que lhe dá a capacidade de captar as ondas sísmicas da terra. Expõem-se assim propostas de criação e expressão artísticas de indivíduos, cujo interesse artístico e capacidade cognitiva pela criação se intensificam, e/ou se serviram destas ferramentas coetâneas para fundamentar outras estéticas e conceitos artísticos oferecendo novos pontos de vista sobre a criação e propondo outras formas de abordar o processo criativo. Isto provoca mudanças no próprio estado da arte propiciando dentro da própria estrutura binária outros suportes de criação para a difusão e para o financiamento.

Pretende-se contribuir para um estudo inovador ao relacionar novos modos de vida no mundo da arte, de expressão e de criatividade para os indivíduos com diversidade funcional, que utilizam novos mecanismos tecnológicos que propiciam e motivam as 
asuas ações e que ao mesmo tempo servem de sistemas de difusão e de financiamento, estratégias transmedia, tanto para a criação como para a comercialização.

Palavras-chave: Arte - Diversidade funcional - Desenho industrial - Era digital - Web 2.0, Processo criativo - Internet - Tecnologia - Ciborgue, Sensores.

[Las traducciones de los abstracts fueron supervisadas por el autor de cada artículo] 